\title{
Prototyped LEACH Routing Algorithm for the Enhancement of Energy Efficiency in Wireless Sensor Network
}

\author{
Neetu Sharma \\ Asst. Prof. \\ Govt. Engineering College, \\ Ajmer
}

\author{
Chandra Prakash Verma \\ Student, M Tech (CS) \\ Govt. Engineering College, \\ Ajmer
}

\author{
Raj Kumar Mahirania \\ Student, M Tech (CS) \\ Govt. Engineering College, \\ Ajmer
}

\begin{abstract}
The topic of discussion and research is wireless sensor network and it just because it is an always application based. The research on wireless sensor networks has recently received much attention by offering an advantage of monitoring different kinds of environments by sensing their physical phenomenon. There are some important parameters are like prolonged network lifetime, scalability, and load balancing for many sensor network applications. Clustering in sensor nodes is an effective technique for achieving efficiency in these parameters. In this work, a prototype energy efficient LEACH algorithm has been written on the basis of energy impact factor for wireless sensor network applications. Low Energy Adaptive Clustering Hierarchy is one of popular cluster-based structures, which has been widely used in wireless sensor networks. To maintain balanced energy consumption LEACH uses TDMA. The prototyped protocol adds feature to LEACH to reduce the consumption of the network resource in each round.
\end{abstract}

\section{Keywords}

WSN, Network life-time, LEACH, clustering, energy impact factor and TDMA

\section{INTRODUCTION}

Radio equipped sensor devices that are autonomously form networks through which sensed data is transported are called wireless sensor networks [1][2]. The applications of wireless sensor networks consists of Military situation, Disaster management, Habitat observing, Medical and health care, Industrial fields, Home networks, Spotting chemical, Biological, radiological, Nuclear, and Explosive material etc. [3].

A wireless sensor network contains hundreds or thousands of these sensor devices that have ability to communicate either directly to the Base Station (BS) or among each other. The nodes in WSNs are usually battery operated sensing devices with limited energy resources because replacing or replenishing the batteries is always not possible or always not an option.

Wireless sensor networks are having some distinguishing differences based on some specific characteristics from mobile ad-hoc networks, cellular networks or wireless networks that's why routing in WSNs is very challenging. Many new algorithms have been proposed, taking into consideration the inherent features of WSNs along with the application and architecture requirements [4].

Clustering schemes offer reduced communication overheads and efficient resource allocations, thus decreasing the overall energy consumption and reducing the interferences among sensor nodes. The basic idea of cluster based routing is to use the information aggregation mechanism and data centric routing in the cluster head to reduce the amount of data transmission and to reduce the energy dissipation in communication and that will achieve the purpose of saving energy of the sensor nodes. That's the main reason of achieving the energy efficiency in cluster based routing in wireless sensor networks. The prototyped algorithm will enhance the energy efficiency of cluster based routing in this type of networks.

"In the clustering routing algorithms for wireless networks, LEACH (low-energy adaptive clustering hierarchy) is considered as the most popular routing protocol that use cluster based routing in order to minimize the energy consumption".

In this paper, there has been proposed an enhancement on the LEACH Protocol that, further enhance the power consumption, in terms of network life time [5].

\section{LITERATURE SURVEY}

The limited energy of sensor nodes has motivated researchers to investigate different schemes to reduce the energy consumption required by routing protocols to deliver messages in WSN[6][7]. Current sensors have the ability of adjusting the power of their radio transmissions [8]. Thus, they can reach their next hop using the minimum amount of energy required for the message to be correctly received by that node. In a WSN, there are situations where a path with a longer hop count can be more energy-efficient than a shorter one.

The energy-aware routing in wireless sensor networks maintains multiple paths and properly chooses one for each packet delivery to improve the network survivability. To get a precise routing metric it may also be quite costly since it needs to exchange routing information very frequently. In a proactive multipath routing algorithm it is provided to achieve energy balance, but it is a load balancing mechanism because of the assumption that "energy burden" and "traffic load" can be assimilated [9].

Clustering in sensor nodes is an effective technique for achieving efficiency in some parameters. Clustering is the method by which sensor nodes in a network organize themselves into hierarchical structures that's why it is called a hierarchical technique. By doing this, sensor nodes can use the network resources such as radio resource and power (energy) more efficiently. When we talk about a cluster data aggregation is performed at cluster head to reduce the data transmission. Cluster formation is usually based on remaining 
energy of sensor nodes and sensor's proximity to cluster-head [9]. Non cluster-head nodes have to choose their cluster-head right after deployment and they all transmit their data to the cluster-head. The role of cluster-head is to forward the data and its own data to the BS after performing data aggregation. LEACH is one of the first hierarchical routing protocols for WSNs [10].

Heinzelman, et.al $[11,12]$ proposed a clustering algorithm for sensor networks, called Low Energy Adaptive Clustering Hierarchy (LEACH).

"LEACH forms clusters by using a distributed algorithm, where nodes make autonomous decisions without any centralized control. LEACH arranges the nodes in the network into clusters and chooses one of them as the cluster head (CH)".

In [13], Zytoune et al. present a Stochastic Low Energy Adaptive Clustering Hierarchy protocol (SLEACH). SLEACH uses the same method proposed in LEACH for forming a cluster. Once the cluster is formed, the cluster head broadcasts in its cluster a data message containing its measurement assuming the pertinent value. Only the nodes, having most significant data, send their messages towards the cluster-head.

In [14], the authors extend SLEACH algorithm by modifying the probability of each node to become cluster-head based on its required energy to transmit to the sink. Their contribution consists in rotation selection of cluster heads considering the remoteness of the nodes to the sink, and the network nodes residual energy.

\section{PROPOSED ALGORITHM}

The idea behind LEACH protocol is to divide the whole wireless sensor networks into clusters. In traditional LEACH always the cluster head node is randomly selected. The opportunity or probability of each node to be selected as cluster head is equal, and energy consumption of the network is averaged. That is the reason why LEACH protocol is used to prolong network life-time.

LEACH algorithm is cyclical in nature; it provides a concept of rounds. LEACH protocol runs the simulation with many rounds.

Each round contains two states:

- Cluster setup state

- Steady state

The algorithm here prototyped is for the resolving the problems that the LEACH algorithm faces. The first problem is that the cluster head in leach algorithm is randomly selected. The proposed scheme is for the selection of cluster head on the basis of two parameters i.e. energy and distance impact factors.

Following is the algorithm that gives the calculation of the energy impact factor:

\subsection{Algorithm (Energy impact factor Calculation)}

1. Each node in cluster checks its own energy (En where $\mathrm{n}$ is no. of nodes in cluster)

2. Ece $=$ Ech, where Ece is minimum value of remaining energy of every node in the cluster and Ech is the energy of current cluster head

3. The current head will receive the energy of each node in cluster
4. Check for each $n$, if (Ece > En)

5. Then Ece $=$ En, $n++$

6. then go to step 4, until the last En received

7. Broadcast Ece to all nodes

8. Found the minimum of En so it will be Ece = $\min (\mathrm{En})$ [Ece is the minimum value of remaining energy of every node in the cluster]

9. Energy impact factor $=(1-($ Ece/En $))$, for each node

In the traditional LEACH algorithm, there are shortcomings. One is, in that algorithm the cluster head is always elected as random and the probability of each node to be elected as cluster head is same. This disadvantage is overcome by this algorithm, because in this algorithm an energy impact factor has been prototyped. After this designing the problem of randomly elections of cluster-head is overcome. The other problem with clustering scheme is to divide the cluster randomly; this shall also be overcome with the algorithm, if efficiently used.

\section{CONCLUSION}

In this paper, a prototyped LEACH algorithm has been given which somewhat resolves energy problems of traditional LEACH algorithm. This is an energy efficient routing protocol which is based on legacy static clustering approach. In this prototyped scheme, new algorithm selection mechanism is inherited from previous LEACH and try to enhance energy consumption by this algorithm whereas; protocol structure is adopted from traditional LEACH protocol. The selected performance metrics are: throughput, energy consumption, number of dead and alive nodes. Therefore, the conclusion is that this extended LEACH is more suitable for homogeneous and heterogeneous WSNs for energy efficiency applications.

\section{REFERENCES}

[1] Akyildiz, W. Su, Y, Sanakara subramaniam,et al, Wireless sensor networks: A survey. Computer Networks.2002,38 (4).

[2] HAN Hong-quan, ZHU Hong-song, MENG Jun, The wireless sensor network. Applications of the Computer Systems, 2005, 8(2).

[3] Kumar, N. Kaur, J, "Improved LEACH Protocol for Wireless Sensor Networks," in Wireless Communications, Networking and Mobile Computing (WiCOM), 2011 7th International Conference on, Sept. 2011.

[4] IJCSI International Journal of Computer Science Issues, Vol. 8, Issue 5, No 1, September 2011 ISSN (Online): 1694-0814

[5] Enhanced LEACH Protocol for Wireless Sensor Networks

[6] W. Heinzelman, A. Chandrakasan and H. Balakrishnan, "Energy-Efficient Communication Protocol for Wireless Micro-sensor Networks," Proceedings of the 33rd Hawaii International Conference on System Sciences (HICSS '00), January 2000.

[7] S. Lindsey, C. Raghavendra, PEGASIS: Power-Efficient Gathering in Sensor Information Systems", IEEE Aerospace Conference Proceedings, 2002, Vol. 3, 9-16 pp. 1125-1130. 
[8] Locally Optimal Source Routing for energy-efficient geographic routing Juan A. Sanchez $Æ$ Pedro M. Ruiz Published online: 1 September 2007 _ Springer Science+ Business Media, LLC 2007

[9] EBRP: Energy-Balanced Routing Protocol for Data Gathering in Wireless Sensor Networks in IEEE Transactions on Parallel And Distributed Systems, VOL. 22, NO. 12, December 2011

[10] Modify LEACH Algorithm for Wireless Sensor Network in IJCSI International Journal of Computer Science Issues, Vol. 8, Issue 5, No 1, September 2011 ISSN (Online): 1694-0814

[11] W.R. Heinzelman, A. Chandrakasan, and H. Balakrishnan, Energy-efficient communication protocol for wireless micro-sensor networks, Proceedings of the 33rd Hawaii International Conference on System Sciences, 2000, 1-10.

[12] W.R. Heinzelman, A. Chandrakasan, and H. Balakrishnan, An Applocation-Specific Protocol Architecture for Wireless Microsensor Networks, IEEE Transactions on Wireless Communications, 2002, 1(4): 662-666.

[13] O. Zytoune, M. El aroussi, M. Rziza, D. Aboutajdine: Stochastic Low Energy Adaptive Clustering Hierarchy, ICGST-CNIR, Volume (8), Issue (1), (2008) pp 4751.

[14] Ouadoudi Zytoune, Youssef Fakhri and Driss Aboutajdine: A Balanced Cost Cluster-Heads Selection Algorithm for Wireless Sensor Networks, International Journal of Computer Science, vol 4, no.1, 2009, pp.2124Enhanced LEACH Protocol for Wireless Sensor Networks 